\title{
Microbiological airway colonization in COPD patients with severe emphysema undergoing endoscopic lung volume reduction
}

This article was published in the following Dove Press journal: International Journal of COPD

\author{
Franziska C Trudzinski' \\ Frederik Seiler' \\ Heinrike Wilkens' \\ Carlos Metz' \\ Annegret Kamp' \\ Robert Bals' \\ Barbara Gärtner ${ }^{2}$ \\ Philipp M Lepper' \\ Sören L Becker ${ }^{2-4}$ \\ 'Department of Internal Medicine V - \\ Pneumology, Allergology and Critical \\ Care Medicine, ECLS Center Saar, \\ University Medical Center Saarland \\ and Saarland University, ${ }^{2}$ Institute of \\ Medical Microbiology and Hygiene, \\ Saarland University, Homburg/Saar, \\ Germany; ${ }^{3}$ Swiss Tropical and Public \\ Health Institute, ${ }^{4}$ University of Basel, \\ Basel, Switzerland
}

Background: Endoscopic lung volume reduction (eLVR) is a therapeutic option for selected patients with COPD and severe emphysema. Infectious exacerbations are serious events in these vulnerable patients; hence, prophylactic antibiotics are often prescribed postinterventionally. However, data on the microbiological airway colonization at the time of eLVR are scarce, and there are no evidence-based recommendations regarding a rational antibiotic regimen.

Objective: The aim of this study was to perform a clinical and microbiological analysis of COPD patients with advanced emphysema undergoing eLVR with endobronchial valves at a single German University hospital, 2012-2017.

Patients and methods: Bronchial aspirates were obtained prior to eLVR and sent for microbiological analysis. Antimicrobial susceptibility testing of bacterial isolates was performed, and pathogen colonization was retrospectively compared with clinical parameters.

Results: At least one potential pathogen was found in 47\% (30/64) of patients. Overall, Gram-negative bacteria constituted the most frequently detected pathogens. The single most prevalent species were Haemophilus influenzae (9\%), Streptococcus pneumoniae (6\%), and Staphylococcus aureus (6\%). No multidrug resistance was observed, and Pseudomonas aeruginosa occurred in $<5 \%$ of samples. Patients without microbiological airway colonization showed more severe airflow limitation, hyperinflation, and chronic hypercapnia compared to those with detected pathogens.

Conclusion: Microbiological airway colonization was frequent in patients undergoing eLVR but not directly associated with poorer functional status. Resistance testing results do not support the routine use of antipseudomonal antibiotics in these patients.

Keywords: COPD, endoscopic lung volume reduction, emphysema, Haemophilus influenzae, Pseudomonas aeruginosa, resistance

\section{Introduction}

Patients with advanced COPD and emphysema are often symptomatic despite intensive pharmacological treatment. Reducing lung volume in hyperinflated COPD patients is an accepted principle to relieve symptoms, as has recently been recommended by the Global Initiative for Chronic Obstructive Lung Disease. ${ }^{1}$ Surgical lung volume reduction has shown to improve exercise capacity, quality of life, and survival in selected patients. ${ }^{2}$ However, the high burden of comorbidities in COPD increases the risk of perioperative morbidity and mortality in this patient population. ${ }^{2,3}$ Consequently, different endoscopic treatment modalities were developed to reduce the invasiveness of this effective approach. ${ }^{4}$ Endoscopic lung volume reduction (eLVR) has become an established treatment for COPD patients with advanced emphysema. ${ }^{1,5}$ During eLVR,
Correspondence: Philipp M Lepper Department of Internal Medicine V Pneumology, Allergology and Critical Care Medicine, ECLS Center Saar, University Medical Center Saarland and Saarland University, Kirrberger Straße, Building 91, 6642I Homburg/Saar, Germany

Tel +49 684I I6 23614

Email philipp.lepper@uks.eu 
endobronchial valves (EBVs), ie, unidirectional duck-bill valves that are designed to induce a complete occlusion and consecutive deflation of the target lobe, are implanted. ${ }^{6}$ While the procedure itself is associated with lower morbidity than surgery, patients undergoing eLVR are at high risk for postinterventional COPD exacerbations. ${ }^{7}$ To prevent such infectious complications, all relevant trials in the field of eLVR with endobronchial valves used a prophylactic antibiotic treatment regimen. However, the antimicrobial substances used differed considerably and included macrolides (eg, azithromycin), second- and third-generation cephalosporins (eg, cefuroxime and cefotaxime), and fluoroquinolones (eg, levofloxacine). ${ }^{7-10}$ A recent "best practice" expert recommendation suggested an empiric, peri-interventional treatment with a broad-spectrum oral antibiotic but did not further specify which substances should be preferably used. ${ }^{11}$

Few studies have characterized the microbiological airway colonization in individuals with advanced lung diseases such as COPD patients referred to eLVR treatment or patients with lung cancer. ${ }^{12}$ Indeed, a recent work pertaining to the role of the microbial flora in these patients revealed a significant microbiological airway colonization but judged that due to "the lack of exhaustive microbiological studies, the conclusions that can be reached remain inconclusive", ie, it remains to be elucidated whether the presence of these pathogens might give rise to infectious complications. ${ }^{12}$ Indeed, it is widely unknown which microbiological organisms might constitute a risk factor if they colonize such patients, and there is an ongoing debate whether infectious exacerbations in COPD patients might actually be caused by those pathogens that previously colonized the patient's airways. Additionally, many COPD patients with advanced emphysema who are eligible for eLVR treatment have had several respiratory infections during their course of disease and might thus have been treated with several antibiotic regimens. It may thus be speculated that higher rates of multiresistant Grampositive (eg, methicillin-resistant Staphylococcus aureus) and particularly Gram-negative pathogens (eg, Pseudomonas aeruginosa) can be detected in these patients. Hence, there is a need to improve the knowledge on the actual microbiological flora in patients undergoing eLVR. Evidence-based recommendations should address 1) whether application of periinterventional antibiotic treatment is justified and 2) which key pathogens should be covered by such a medication.

Here, we present a single-center study reporting on the microbiological airway colonization and antibiotic susceptibility patterns of potential pathogens isolated from COPD patients with severe emphysema undergoing eLVR in one
University hospital in southwest Germany, and we discuss the arising implications for the choice of periinterventional antibiotic treatment.

\section{Patients and methods Study site and patient selection}

This study is a retrospective analysis of consecutive patients treated at the Saarland University Medical Center in Homburg, Germany, between March 2012 and March 2017. All patients who underwent eLVR with endobronchial valve implantation were retrieved from an electronic database. The procedure was standardized by an institutional protocol during the study period. Advanced COPD patients were eligible for this treatment modality if they were highly symptomatic, despite an established pharmacological combination treatment consisting of long-acting beta agonists, long-acting muscarinic antagonists, and inhaled corticosteroids. Furthermore, patients had to quit smoking for at least 3 months prior to the intervention. On admission for intervention, acute exacerbation or respiratory infection was excluded clinically and by laboratory tests. Postinterventionally, all patients completed a 5-day course of oral antibiotic treatment (sultamicillin $375 \mathrm{mg}$ twice a day) and $50 \mathrm{mg}$ oral prednisolone. The study was approved by the institutional review board of Ärztekammer des Saarlandes; No 35/15. The necessity for informed consent was waived by the institutional review board due to the retrospective nature of the study.

\section{Characteristics of endoscopic and periinterventional procedures}

Bronchoscopy and periprocedural preparations were performed according to the institutional standard. Pulmonary function tests were performed with a Jaeger MasterScreen Body System (CareFusion, Rolle, Switzerland); static lung volumes were determined by whole-body plethysmography. All measurements were performed according to the American Thoracic Society (ATS) and the European Respiratory Society (ERS) guidelines. ${ }^{13,14}$ The 6-minute walking test (6MWT) was measured in all patients on a $70 \mathrm{~m}$ floor after providing standard instructions, while supplemental oxygen was administered through a nasal cannula as needed to maintain the arterial oxygen saturation at $\geq 90 \%{ }^{15}$

Endoscopic interventions were performed under total intravenous anesthesia. The patients were intubated and mechanically ventilated. Bronchial aspirates were taken prior to the intervention and were immediately sent to the microbiology laboratory. EBVs (Zephyr EBV; Pulmonx, Redwood City, CA, USA) were placed unilaterally in lobar, 
segmental, or subsegmental bronchi based on the individual anatomic conditions with the intention of completely isolating the target lobe. All patients underwent a chest X-ray 1 hour after the procedure (or earlier if symptoms occurred) to exclude a pneumothorax.

\section{Processing of bronchial aspirates for microbiological analysis}

Endobronchial aspirates obtained during eLVR were immediately sent via a pneumatic transport system to the microbiology laboratory for same-day processing. Gram-stained microscope slides were prepared from each specimen, and samples were plated on different solid agar media to detect bacteria and fungi, ie, blood agar, MacConkey agar, chocolate agar, and Sabouraud agar. For the detection of mycobacteria, Auramine staining was performed and mycobacterial growth indicator tube (MGIT) liquid culture as well as LoewensteinJensen agar and Stonebrink agar were employed. In addition, if requested by the clinician, a polymerase chain reaction assay for the detection of Bordetella pertussis, Chlamydophila pneumoniae, Haemophilus influenzae, Legionella pneumophila, Mycoplasma pneumoniae, and Streptococcus pneumoniae was carried out. For the detection of bacteria, fungi, and mycobacteria, samples were incubated for 48-72 hours, 3 weeks, and 12 weeks, respectively, and were regularly examined for growth. Matrix-assisted laser desorption ionization time-of-flight (MALDI-TOF) mass spectrometry was carried out for species-specific identification of bacteria and fungi.

\section{Antibiotic resistance testing}

Culture-grown colonies of bacteria were subjected to automated antibiotic susceptibility testing using the VITEK2 system (BioMérieux, Marcy-l'Étoile, France). In case of remarkably resistant strains and/or implausible results, the Etest method was used on Mueller-Hinton agar to determine the specific minimal inhibitory concentrations (MICs) of the tested bacterial species. Breakpoints defined by the European Committee on Antimicrobial Susceptibility Testing (EUCAST) were used for interpretation as sensitive, intermediately sensitive, and resistant. ${ }^{16}$

\section{Statistical analysis}

Statistical analysis was performed using SPSS Version 21 (IBM Corporation, Armonk NY, USA). Data were analyzed using Fisher's exact test or $\chi^{2}$ test, as appropriate, for categorical variables and using a two-sided $t$-test for independent samples for continuous variables. A $P$-value of $\leq 0.05$ was considered as statistically significant.

\section{Results Microbiological airway colonization}

Bronchial aspirates stemming from 64 patients were obtained during eLVR and sent for microbiological examination. No microbial organism was detected in 34 individuals (53\%), whereas at least one potential pathogen was found in 30 patients, owing to a bronchial aspirate positivity rate of $47 \%$. Bacteria accounted for the majority of positive specimens $(23 / 30 ; 77 \%)$, and 14 different species were detected in the patient samples, 10 of which were Gram-negative. The most prevalent bacteria were $H$. influenzae $(\mathrm{n}=6 ; 9 \%)$, $S$. pneumoniae $(\mathrm{n}=4 ; 6 \%)$, and $S$. aureus $(\mathrm{n}=4 ; 6 \%)$. The non-fermentative Gram-negative bacterium $P$. aeruginosa was detected in $4.7 \%$ of the analyzed samples. Fungi were found in $13 \%$ of all specimens, and these were mainly yeasts. No mycobacteria were found in the cohort. A total of $16 \%$ of all samples grew more than one organism, but no more than three organisms were detected concurrently in one sample. Details on the occurrence of bacteria and fungi are displayed in Table 1.

Table I Microbiological characterization of bacterial and fungal lower airway colonization of 64 COPD patients undergoing endoscopic lung volume reduction for advanced emphysema in Homburg, Germany, between March 2012 and March 2017

\begin{tabular}{|c|c|c|}
\hline \multirow[t]{2}{*}{ Pathogen } & \multicolumn{2}{|c|}{ Total $(n=64)$} \\
\hline & n & $\%$ \\
\hline Total number of positive samples & $30^{\mathrm{a}}$ & 47 \\
\hline Bacteria & $23^{\mathrm{a}}$ & 36 \\
\hline Gram-positive bacteria & 10 & 16 \\
\hline Staphylococcus aureus & 4 & 6 \\
\hline Streptococcus constellatus & 1 & 2 \\
\hline Streptococcus pneumoniae & 4 & 6 \\
\hline Streptococcus pyogenes & 1 & 2 \\
\hline Gram-negative bacteria & $17^{\mathrm{a}}$ & 27 \\
\hline Achromobacter xylosoxidans & 1 & 2 \\
\hline Bordetella bronchiseptica & I & 2 \\
\hline Enterobacter cloacae complex & I & 2 \\
\hline Haemophilus influenzae & 6 & 9 \\
\hline Haemophilus parainfluenzae & 2 & 3 \\
\hline Klebsiella pneumoniae & 2 & 3 \\
\hline Moraxella catarrhalis & I & 2 \\
\hline Pseudomonas aeruginosa & 3 & 5 \\
\hline Serratia marcescens & 2 & 3 \\
\hline Stenotrophomonas maltophilia & 1 & 2 \\
\hline Fungi & $8^{a}$ & 13 \\
\hline Yeasts & 6 & 9 \\
\hline Candida albicans & 5 & 6 \\
\hline Candida glabrate & 3 & 5 \\
\hline Candida krusei & I & 2 \\
\hline Molds & 2 & 3 \\
\hline Aspergillus terreus & I & 2 \\
\hline Penicillium spp. & I & 2 \\
\hline
\end{tabular}

Note: ${ }^{a}$ Cocolonization with different organisms was found in some patients. 


\section{Patient characteristics}

All 64 patients presented with severe airflow obstruction and hyperinflation. The patient characteristics were comparatively stratified by individuals with and without detectable microbiological airway colonization (Table 2). Those patients without airway colonization showed significantly lower values for forced expiratory volume in 1 second $\left(\mathrm{FEV}_{1}\right)$ compared to those with the detection of at least one potential pathogen ( $0.62 \pm 0.18$ versus $0.80 \pm 0.37 \mathrm{~L}$, equaling $24.81 \pm 7.02$ versus $30.01 \% \pm 11.35 \%$ of predicted). Hyperinflation shown by the residual volume-to-total lung capacity (RV/TLC) ratio was more pronounced in patients without detection of microbio-

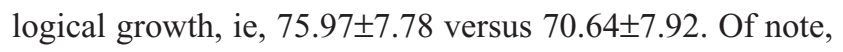
the noncolonized patients presented also more frequently with hypercapnia (44 versus $10 \% ; P=0.002$ ).

\section{Antibiotic susceptibility patterns}

Among the 10 isolated Gram-positive bacteria strains, resistance to penicillin was observed in one isolate of S. aureus, whereas all streptococci were sensitive to penicillin. Three $S$. aureus strains were resistant to macrolides, but all Grampositive pathogens were sensitive to ampicillin/sulbactam. Among Gram-negative pathogens, all $H$. influenzae strains showed reduced susceptibility to macrolides and two of the six strains were resistant to ampicillin/sulbactam, whereas all isolates were sensitive to the third-generation cephalosporin cefotaxime. One strain of $P$. aeruginosa displayed resistance to piperacillin/tazobactam, fluoroquinolones, and aminoglycosides but remained sensitive to ceftazidime and meropenem. Among all Gram-negative bacteria, no extended spectrum beta-lactamase-producing species were detected and multidrug resistance was not observed. Within the Enterobacteriaceae, resistance to ampicillin/sulbactam was exclusively observed in two Serratia marcescens strains, while all species remained sensitive to piperacillin/tazobactam, cefotaxime, ceftazidime, ciprofloxacin, gentamicin, and meropenem. The antimicrobial susceptibility patterns of frequently encountered Gram-positive and Gram-negative bacteria are summarized in Table 3 .

\section{Discussion}

In our cohort of patients undergoing eLVR, airway colonization with different microbiological organisms was detected in half of all individuals. The most prevalent bacteria were $H$. influenzae, S. pneumoniae, and $S$. aureus. Colonization with $P$. aeruginosa occurred in only $4.7 \%$ of all samples. Of note, the antimicrobial susceptibility patterns did not yield significant rates of multidrug-resistant pathogens, despite the patients' advanced pulmonary impairment and frequent previous anti-infective treatment.

COPD exacerbations are defined as an acute worsening of respiratory symptoms that requires additional treatment.

Table 2 Comparison of baseline characteristics in patients undergoing endoscopic lung volume reduction in Homburg, Germany (2012-2017), stratified by microbiological airway colonization

\begin{tabular}{|c|c|c|c|c|}
\hline Clinical presentation & All patients, $N=64$ & Not colonized, $\mathbf{N}=\mathbf{3 4}$ & Colonized, $\mathbf{N}=\mathbf{3 0}$ & $P$-value \\
\hline Age (years) & $62.4 I \pm 8.67$ & $62.28 \pm 8.25$ & $62.56 \pm 9.26$ & 0.901 \\
\hline Male $(\mathrm{N})$ & $32 / 64(50)$ & $\mid 4 / 34(4 \mid)$ & $18 / 30(60)$ & 0.210 \\
\hline Height (m) & $1.66 \pm 0.10$ & $1.65 \pm 0.11$ & $1.67 \pm 0.10$ & 0.680 \\
\hline Weight (kg) & $62.29 \pm 14.88$ & $59.91 \pm 13.70$ & $64.99 \pm|5.9|$ & 0.180 \\
\hline BMI $\left(\mathrm{kg} / \mathrm{m}^{2}\right)$ & $22.52 \pm 4.50$ & $21.81 \pm 4.29$ & $23.32 \pm 4.66$ & 0.184 \\
\hline 6MWT $(m)^{\mathrm{a}}$ & $259.37 \pm 109.69$ & $248.58 \pm 99.44$ & $27 \mid .66 \pm 120.91$ & 0.419 \\
\hline \multicolumn{5}{|l|}{ Blood gas analysis } \\
\hline $\mathrm{PaO}_{2}<55 \mathrm{mmHg}$ and/or LTO & $48 / 64(75)$ & $26 / 34(76)$ & $22 / 30(73)$ & 0.781 \\
\hline $\mathrm{PaCO}_{2}>50 \mathrm{mmHg}$ & I8/64 (28) & I5/34 (44) & $3 / 30(10)$ & $0.002 *$ \\
\hline \multicolumn{5}{|l|}{ Baseline lung function } \\
\hline $\mathrm{FEV}_{1}(\mathrm{~L})$ & $0.70 \pm 0.30$ & $0.62 \pm 0.18$ & $0.80 \pm 0.37$ & $0.022 *$ \\
\hline $\mathrm{FEV}_{1}(\%)$ & $27.25 \pm 9.50$ & $24.8 \mathrm{I} \pm 7.02$ & $30.01 \pm 11.35$ & $0.035 *$ \\
\hline $\mathrm{VC}(\mathrm{L})$ & $2.11 \pm 0.87$ & $1.95 \pm 0.79$ & $2.29 \pm 0.93$ & 0.132 \\
\hline VC (\%) & $62.72 \pm 20.11$ & $59.81 \pm 20.37$ & $66.03 \pm 19.62$ & 0.219 \\
\hline $\mathrm{RV}(\mathrm{L})$ & $5.86 \pm 1.33$ & $6.09 \pm 1.41$ & $5.59 \pm 1.20$ & 0.136 \\
\hline $\mathrm{RV}(\%)$ & $27 I .7 I \pm 72.04$ & $284.70 \pm 59.78$ & $256.98 \pm 82.37$ & 0.134 \\
\hline TLC (L) & $7.91 \pm 1.61$ & $7.99 \pm 1.76$ & $7.83 \pm 1.46$ & 0.703 \\
\hline TLC (\%) & $|40.09 \pm 2| .30$ & $143.69 \pm 18.87$ & $136.02 \pm 23.42$ & 0.158 \\
\hline $\mathrm{RV} / \mathrm{TLC}$ & $73.47 \pm 8.23$ & $75.97 \pm 7.78$ & $70.64 \pm 7.92$ & $0.009 *$ \\
\hline
\end{tabular}

Notes: Values are presented as $\mathrm{n}(\%)$ or mean $\pm \mathrm{SD}$. ${ }^{*} \mathrm{P} \leq 0.05$ was considered statistically significant. ${ }^{2} 6 \mathrm{MWT}$ results were only available for 62 patients.

Abbreviations: $\mathrm{BMI}$, body mass index; $\mathrm{FEV}_{1}$, forced expiratory volume in I second; LTOT, long-term oxygen therapy; 6MWT, 6-minute walking test; PaCO ${ }_{2}$, partial pressure of carbon dioxide; $\mathrm{PaO}_{2}$, partial pressure of oxygen; $\mathrm{RV}$, residual volume; TLC, total lung capacity; VC, vital capacity. 


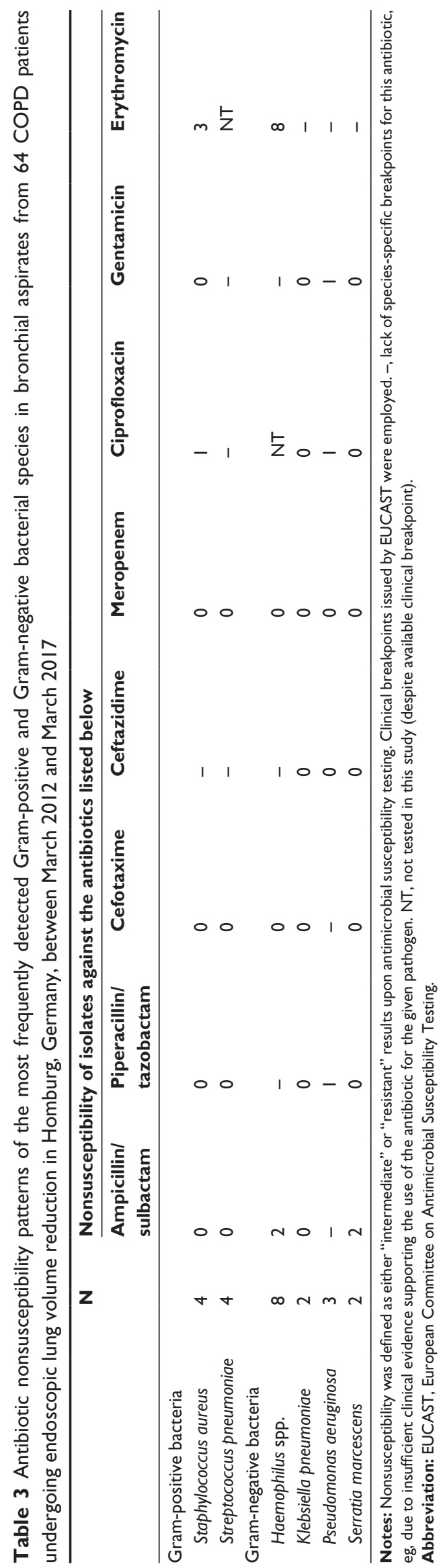

These events are of high prognostic relevance and impact on the quality of life. ${ }^{1}$ Especially patients with advanced emphysema are prone to exacerbations following bronchoscopy, even independent of valve placement. Indeed, in the BeLieVeR-HIFi trial, Davey et al ${ }^{10}$ reported that $20(80 \%)$ of the 25 patients in the control group developed mild-to-severe exacerbations following bronchoscopy with sham valve placement. Generally, manifest acute bronchitis, pneumonia, and/or lung infections within the first 3 months following EBV treatment are reported in up to $20 \%$ of individuals who underwent eLVR. ${ }^{11}$ Hence, most interventionists use prophylactic antibiotic and anti-inflammatory treatment to prevent postinterventional exacerbations. However, there is no consensus regarding the choice of a rational antibiotic regimen, which can mainly be explained by the lack of microbiological studies examining the airway colonization of pneumological patients who are referred to eLVR treatment. ${ }^{17,18}$ While it is not fully understood whether airway colonization with respiratory pathogens increases the risk to develop infections caused by the same agents, colonization with multiresistant or difficult-to-treat bacteria such as $P$. aeruginosa is acknowledged to worsen the course of disease. ${ }^{19,20}$ In addition, recent research indicates that chronically colonized COPD patients may have a worse clinical course due to the development of deleterious immune responses. ${ }^{21}$ Therefore, it has been recommended to prescribe "prophylactic" antibiotics at least in the subgroup of patients who have had previous colonization or infections with pathogenic bacteria. ${ }^{22}$

In our study, $H$. influenzae and Gram-positive cocci constituted the most frequently encountered pathogen species, whereas multidrug resistance was not common. $P$. aeruginosa and other intrinsically resistant Gram-negative pathogens such as Achromobacter xylosoxidans and Stenotrophomonas maltophilia were rarely encountered. Hence, a postinterventional antibiotic treatment with the beta-lactam/ beta-lactamase inhibitor sultamicillin (an oral formulation of ampicillin/sulbactam) would have covered virtually $100 \%$ of Gram-positive pathogens, $75 \%$ of Haemophilus spp., and many of the remaining Gram-negative bacteria. Of note, the second-generation cephalosporin cefuroxime would have reached a similar in vitro efficacy. However, macrolide antibiotics (ie, erythromycin, clarithromycin, and azithromycin), which are commonly prescribed for respiratory infections because of their additional immunomodulatory properties, ${ }^{23}$ would have had significant less activity, which can mainly be attributed to the low observed susceptibility of $H$. influenzae and $S$. aureus to erythromycin. In addition, macrolide activity against $S$. pneumoniae has considerably 
decreased in Europe, North America, and elsewhere. ${ }^{24,25}$ The aforementioned pathogens are of particular relevance in COPD patients, in whom they are strikingly prevalent, as has recently been confirmed by a study on the etiology of community-acquired pneumonia in Germany. ${ }^{26}$ Indeed, $H$. influenzae is now recognized as one key pathogen that induces significant inflammation in the airways of COPD patients, ${ }^{27}$ and it should thus be covered by any antibiotic administered to patients undergoing eLVR. While azithromycin has been shown to reduce the frequency of exacerbations in patients with $\mathrm{COPD},{ }^{28}$ its antimicrobial activity to effectively clear $H$. influenzae may be insufficient, and long-term treatment with this agent might lead to increased macrolide resistance of bacteria colonizing the airways of COPD patients. ${ }^{29}$ Hence, in agreement with the findings from our study, the use of a beta-lactam antibiotic might be advantageous if compared with macrolides. In addition, we did not find high rates of multiresistant Gram-negative pathogens, so that the use of antipseudomonal agents would not have been advisable.

Interestingly, microbiological airway colonization was associated with a better functional status in our study, the reason for which remains unclear. Yet, it might be hypothesized that colonized patients were more symptomatic, eg, due to recurrent or more frequent exacerbations, ${ }^{30}$ and might thus have sought additional treatment options such as eLVR earlier than noncolonized patients. While it has been shown repeatedly that some bacterial species (eg, $P$. aeruginosa) negatively impact on COPD patients, ${ }^{16-18}$ there is insufficient evidence to conclude that bacterial colonization per se worsens the course of the disease. Hence, further studies with a larger sample size are warranted to elucidate whether a pathogen-specific association between airway colonization and clinical signs can be observed.

Our study has limitations that need to be addressed. First, the interventional procedures were performed according to standardized operating procedures, but the analysis was carried out retrospectively and our data stem from a limited number of patients treated at a single center, so that not all potential confounders (eg, frequency of previous antibiotic treatment) could be systematically assessed, and our results may thus not be uncritically generalized to all patients undergoing eLVR. Second, we cannot fully exclude that the patients without airway colonization had more recently received antibiotic treatment. Yet, it is unlikely that anti-infective medication was prescribed at least during the last weeks before eLVR, because this procedure was exclusively performed in stable COPD patients without an active exacerbation. Third, the sensitivity of the employed molecular and culture-based diagnostic tests might have been further enhanced by the application of additional molecular, sequencing-based techniques, which would have allowed a more precise characterization of the bronchial microbiome. Fourth, obtaining bronchial aspirates is more prone to contamination by upper airway flora than, eg, samples stemming from bronchoalveolar lavages or protected specimen brushes. Additional studies are thus warranted to further explore the associations between the airway colonization of eLVR patients and related infectious complications. Finally, it is important to note that in vitro susceptibility profiles do not necessarily predict clinical efficacy and should thus be interpreted with caution.

\section{Conclusion}

Microbiological airway colonization was frequent in patients undergoing eLVR. Our findings suggest that postinterventional treatment of these patients with an oral beta-lactam/ beta-lactamase inhibitor (eg, sultamicillin) or a secondgeneration cephalosporin (eg, cefuroxime) would have covered most pathogens, and these were in vitro more active than macrolides. There is an urgent need for additional studies elucidating the clinical significance of microbiological airway colonization in COPD patients being treated with eLVR.

\section{Disclosure}

The authors report no conflicts of interest in this work.

\section{References}

1. Vogelmeier CF, Criner GJ, Martinez FJ, et al. Global strategy for the diagnosis, management, and prevention of chronic obstructive lung disease 2017 report. GOLD executive summary. Am J Respir Crit Care Med. 2017;195(5):557-582.

2. Fishman A, Martinez F, Naunheim K, et al. A randomized trial comparing lung-volume-reduction surgery with medical therapy for severe emphysema. N Engl J Med. 2003;348(21):2059-2073.

3. Naunheim KS, Wood DE, Krasna MJ, et al. Predictors of operative mortality and cardiopulmonary morbidity in the National Emphysema Treatment Trial. J Thorac Cardiovasc Surg. 2006;131(1):43-53.

4. Venuta F, Rendina EA, De Giacomo T, et al. Bronchoscopic procedures for emphysema treatment. Eur J Cardiothorac Surg. 2006;29(3): 281-287.

5. Herth FJF, Slebos DJ, Criner GJ, Shah PL. Endoscopic lung volume reduction: an expert panel recommendation - update 2017. Respiration. 2017;94(4):380-388.

6. Herth FJ, Noppen M, Valipour A, et al. Efficacy predictors of lung volume reduction with Zephyr valves in a European cohort. Eur Respir J. 2012; 39(6):1334-1342.

7. Sciurba FC, Ernst A, Herth FJ, et al. A randomized study of endobronchial valves for advanced emphysema. N Engl J Med. 2010;363(13): 1233-1244.

8. Klooster K, ten Hacken NH, Hartman JE, Kerstjens HA, van Rikxoort EM, Slebos DJ. Endobronchial valves for emphysema without interlobar collateral ventilation. $N$ Engl J Med. 2015;373(24):2325-2335. 
9. Valipour A, Slebos DJ, Herth F, et al. Endobronchial valve therapy in patients with homogeneous emphysema. Results from the IMPACT Study. Am J Respir Crit Care Med. 2016;194(9):1073-1082.

10. Davey C, Zoumot Z, Jordan S, et al. Bronchoscopic lung volume reduction with endobronchial valves for patients with heterogeneous emphysema and intact interlobar fissures (the BeLieVeR-HIFi study): a randomised controlled trial. Lancet. 2015;386(9998):1066-1073.

11. Slebos DJ, Shah PL, Herth FJ, Valipour A. Endobronchial valves for endoscopic lung volume reduction: best practice recommendations from expert panel on endoscopic lung volume reduction. Respiration. 2017; 93(2):138-150.

12. D'Journo XB, Rolain JM, Doddoli C, Raoult D, Thomas PA. Airways colonizations in patients undergoing lung cancer surgery. Eur $J$ Cardiothorac Surg. 2011;40(2):309-319.

13. Miller MR, Hankinson J, Brusasco V, et al. Standardisation of spirometry. Eur Respir J. 2005;26(2):319-338.

14. Miller MR, Crapo R, Hankinson J, et al. General considerations for lung function testing. Eur Respir J. 2005;26(1):153-161.

15. ATS Committee on Proficiency Standards for Clinical Pulmonary Function Laboratories. ATS statement: guidelines for the six-minute walk test. Am J Respir Crit Care Med. 2002;166(1):111-117.

16. European Committee on Antimicrobial Susceptibility Testing (EUCAST). Breakpoint Tables for Interpretation of MICs and Zone Diameters, Version 7.1; 2017. Available from: http://www.eucast.org/fileadmin/src/media/ PDFs/EUCAST_files/Breakpoint_tables/v_7.1_Breakpoint_Tables.pdf. Accessed April 25, 2017.

17. Siegel SJ, Weiser JN. Mechanisms of bacterial colonization of the respiratory tract. Annu Rev Microbiol. 2015;69:425-444.

18. Patel IS, Seemungal TA, Wilks M, Lloyd-Owen SJ, Donaldson GC, Wedzicha JA. Relationship between bacterial colonisation and the frequency, character, and severity of COPD exacerbations. Thorax. 2002;57(9):759-764.

19. Nseir S, Ader F, Lubret R, Marquette CH. Pathophysiology of airway colonization in critically ill COPD patient. Curr Drug Targets. 2011; 12(4):514-520.
20. Matkovic Z, Miravitlles M. Chronic bronchial infection in COPD. Is there an infective phenotype? Respir Med. 2013;107(1):10-22.

21. Leung JM, Tiew PY, Mac Aogain M, et al. The role of acute and chronic respiratory colonization and infections in the pathogenesis of COPD. Respirology. 2017;22(4):634-650.

22. Casutt A, Koutsokera A, Lovis A. Lung volume reduction coil treatment: is there an indication for antibiotic prophylaxis? Arch Bronconeumol. 2016;52(2):114-115.

23. Qiu S, Zhong X. Macrolides: a promising pharmacologic therapy for chronic obstructive pulmonary disease. Ther Adv Respir Dis. 2017;11(3): $147-155$.

24. Hicks LA, Monnet DL, Roberts RM. Increase in pneumococcus macrolide resistance, USA. Emerg Infect Dis. 2010;16(5):896-897.

25. Cherazard R, Epstein M, Doan TL, Salim T, Bharti S, Smith MA. Antimicrobial resistant Streptococcus pneumoniae: prevalence, mechanisms, and clinical implications. Am J Ther. 2017;24(3):e361-e369.

26. Braeken DC, Franssen FM, von Baum H, et al. Bacterial aetiology and mortality in COPD patients with CAP: results from the German Competence Network, CAPNETZ. Int J Tuberc Lung Dis. 2017;21(2): 236-243.

27. Wu D, Hou C, Li Y, et al. Analysis of the bacterial community in chronic obstructive pulmonary disease sputum samples by denaturing gradient gel electrophoresis and real-time PCR. BMC Pulm Med. 2014;14:179.

28. Ni W, Shao X, Cai X, et al. Prophylactic use of macrolide antibiotics for the prevention of chronic obstructive pulmonary disease exacerbation: a meta-analysis. PLoS One. 2015;10(3):e0121257.

29. Pettigrew MM, Tsuji BT, Gent JF, et al. Effect of fluoroquinolones and macrolides on eradication and resistance of Haemophilus influenzae in chronic obstructive pulmonary disease. Antimicrob Agents Chemother. 2016;60(7):4151-4158.

30. Fuschillo S, Martucci M, Donner CF, Balzano G. Airway bacterial colonization: the missing link between COPD and cardiovascular events? Respir Med. 2012;106(7):915-923.
International Journal of COPD

\section{Publish your work in this journal}

The International Journal of COPD is an international, peer-reviewed journal of therapeutics and pharmacology focusing on concise rapid reporting of clinical studies and reviews in COPD. Special focus is given to the pathophysiological processes underlying the disease, intervention programs, patient focused education, and self management protocols.

\section{Dovepress}

This journal is indexed on PubMed Central, MedLine and CAS. The manuscript management system is completely online and includes a very quick and fair peer-review system, which is all easy to use. Visit http://www.dovepress.com/testimonials.php to read real quotes from published authors. 